\title{
Regularization of fields for self-force problems in curved spacetime: foundations and a time-domain application
}

\author{
Ian Vega and Steven Detweiler \\ Institute for Fundamental Theory, Department of Physics, \\ University of Florida, Gainesville, FL 32611-8440
}

(Dated: January 15, 2008)

\begin{abstract}
We propose an approach for the calculation of self-forces, energy fluxes and waveforms arising from moving point charges in curved spacetimes. As opposed to mode-sum schemes that regularize the self-force derived from the singular retarded field, this approach regularizes the retarded field itself. The singular part of the retarded field is first analytically identified and removed, yielding a finite, differentiable remainder from which the self-force is easily calculated. This regular remainder solves a wave equation which enjoys the benefit of having a non-singular source. Solving this wave equation for the remainder completely avoids the calculation of the singular retarded field along with the attendant difficulties associated with numerically modeling a delta function source. From this differentiable remainder one may compute the self-force, the energy flux, and also a waveform which reflects the effects of the self-force.

As a test of principle, we implement this method using a 4th-order $(1+1)$ code, and calculate the self-force for the simple case of a scalar charge moving in a circular orbit around a Schwarzschild black hole. We achieve agreement with frequency-domain results to $\sim 0.1 \%$ or better.

PACS numbers: 04.25.D-, 04.25.dg, 04.25.Nx, 04.20.Cv, 04.30.Db
\end{abstract}

\section{INTRODUCTION}

With the advent of gravitational wave astronomy approaching, the development of accurate and efficient models for gravitational wave sources has steadily progressed. The ability to predict gravitational wave amplitudes and waveforms for expected sources will greatly enhance the usefulness of detectors such as VIRGO, LIGO, and LISA. An interesting class of relevant sources includes a large $10^{2}-10^{10} \mathrm{M}_{\odot}$ black hole in a binary with a closely orbiting stellar-mass compact object.

The orbit and inspiral of a compact object into a substantially more massive black hole presents a complication for traditional numerical analysis. A numerical grid must be fine enough to resolve the geometry in the vicinity of the small object, where the metric appears to be that of the compact object with tidal distortions from the large hole. But the grid must be coarse enough to reach the wave-zone of the binary, so that the waveforms might be carefully monitored. In addition, the timescale for the effect of radiation reaction is long compared with the orbital period and the waveform from many orbits will be used in the data analysis. Together the dramatically different length scales coupled with the dramatically different time scales present a formidable challenge for the study of an extreme mass ratio inspiral (EMRI).

Perturbative analysis appears more feasible for the EMRI problem. The orbiting compact object is modeled as a point mass whose motion, to lowest-order, approximately follows a geodesic in the background geometry of the large black hole companion. Thus, the emitted grav-

*Electronic address: vega@phys.ufl.edu itational waves can be calculated reasonably well using the mature perturbation theory of black holes [1, 2, , 3]. But in this approach it is necessary to go beyond the approximation of geodesic motion in the background geometry. The effects of radiation reaction on the orbital phase requires an extension of the usual perturbation analysis to include what is often called the self-force.

In a so-called frequency-domain approach, one chooses to Fourier decompose the source and the field and then solves for each Fourier mode of the field independently. This method works well for a flux calculation if the spectrum is simple, such as that of a particle in a circular orbit. However for generic trajectories, including particularly those which reflect the effects of radiation reaction, the frequency spectrum is complicated enough to make the frequency-domain analysis numerically expensive.

Further, the field of the particle is singular at the particle's location, and some regularization procedure is required to calculate self-force effects. The usual procedure to date is termed mode-sum regularization, as initially developed by Barack and Ori [4, 5, 6]. This regularization prescription depends crucially upon a decomposition of the derivatives of the field into angular modes, such as spherical harmonics, which are individually finite. From each finite mode, the part that contributes to the singularity but not the self-force is identified and removed; the remainders of the field derivatives for each mode are then summed to determine the finite effect of the particle's field on its own motion. Generally the sum has convergence which is only polynomial in the mode number and, thus, requires analysis at high mode numbers [7, 8, 9, 10, 11, 12, 13, 14, 15] to obtain accurate results.

In this manuscript we introduce a general method for analyzing the field of a point charge orbiting a black hole and for directly determining the waveforms and flux in- 
tegrals as well as the instantaneous self-force acting back on the charge itself, which includes all of the effects of radiation reaction in a natural manner.

The strength of this approach lies in the derivation of a wave equation for a regular field $\psi^{R}$ which is identical to the retarded field in the wave zone and whose derivatives at the charge determine the self-force. We call the determination of $\psi^{R}$, field regularization. Solving our effective wave equation requires neither Fourier decomposition in time, nor any angular decomposition for treating the dramatically different length scales in the EMRI problem, and circumvents the need for ever calculating the actual singular retarded field.

The source $S_{\text {eff }}$ of the effective wave equation follows from a local analysis of the singular part $\psi^{S}$ of the retarded field, Eq. (3). Importantly, this effective source is smooth everywhere except for its limited differentiability at the location of the charge. Ample freedom in choosing $S_{\text {eff }}$ allows the source to spread out over a region with a length scale comparable to the size of the black hole or even to the distance from the charge to the black hole.

We shall describe our approach in terms of a point source with a scalar charge interacting with its own scalar field while orbiting a large black hole. The formal extension of these ideas to pure gravity with a small Schwarzschild black hole perturbing the geometry of a much larger black hole is completely straightforward at the perturbative level. The details of this extension to gravity are algebraically complicated but conceptually simple and will be the focus of a future report.

\section{ORGANIZATION OF THIS PAPER}

The main objective of this work is to provide a proof of principle for the process of field regularization described in $₫ \amalg I I$ as a time-domain technique for self-force calculation. It verifies that we are able to achieve results comparable to that obtained with frequency-domain methods [9], or other time-domain methods relying on the modesum decomposition [13, 14].

In $₫ \mathrm{IV}$ we describe the details of our numerical implementation of field regularization applied to a scalar charge in a circular orbit in Schwarzschild. Tests of the internal consistency of the numerical implementation are in $8 \mathrm{~V}$.

Section VI displays the results of our self-force analysis for a scalar charge in circular orbits at Schwarzschild radius $R=10 M$ and $12 M$. The time and radial components of the self force are compared with results from a frequency-domain analysis. We also reconstruct the entire retarded field and compare this with the retarded field of the frequency domain analysis.

The discussion in \$VII summarizes our results and describes the strengths of field regularization in comparison with other methods of self-force calculation and also with methods of current interest for calculating energy fluxes and waveforms for generic orbits about black holes.
Appendix $\mathrm{A}$ gives some details of the expansion of the singular field $\psi^{S}$ about the point charge and describes how higher order terms in the expansion increase the overall efficiency of field regularization.

\section{FIELD REGULARIZATION}

For a scalar charge, the general strategy for computing the self-force first involves solving the minimally-coupled scalar wave equation with a point charge $q$ source,

$$
\nabla^{a} \nabla_{a} \psi^{\mathrm{ret}}=-4 \pi q \int_{\gamma} \delta^{(4)}(x-z(\tau)) d \tau
$$

for the retarded field $\psi^{\text {ret }}$. Here $\nabla_{a}$ is the derivative operator associated with the metric $g_{a b}$ of the background spacetime and $\gamma$ is the worldline of the charge defined by $z^{a}(\tau)$ and parameterized by the proper time $\tau$. The physical solution of the resulting wave equation will be a retarded field that is singular at the location of the point charge. As such, a self-force naively expressed as

$$
F_{a}(\tau)=q \nabla_{a} \psi^{\mathrm{ret}}(z(\tau))
$$

will need a regularization prescription to make sense. Early regularization prescriptions [16, 17, 18] were based upon a Hadamard expansion of the Green function, and showed that for a particle moving along a geodesic the self force could be described in terms of the particle interacting only with the "tail" part of $\psi$, which is finite at the particle itself. Later [19] it was realized that the singular part of the field $\psi^{S}$ which exerts no force on the particle itself could be identified as an actual solution to Eq. (11) in a neighborhood of the particle. A formal description of $\psi^{S}$ in terms of parts of the retarded Green's function [19] is possible, but generally there is no exact functional description for $\psi^{S}$ in a neighborhood of the particle. Fortunately, an intuitively satisfying description for $\psi^{S}$ results from a careful expansion about the location of the particle:

$$
\psi^{S}=q / \rho+O\left(\rho^{3} / \mathcal{R}^{4}\right) \text { as } \rho \rightarrow 0,
$$

where $\mathcal{R}$ is a constant length scale of the background geometry and $\rho$ is a scalar field which simply satisfies $\rho^{2}=x^{2}+y^{2}+z^{2}$ in a very special Minkowskii-like locally inertial coordinate system centered on the particle, first described by Thorne, Hartle and Zhang 20, 21] and applied to self-force problems in Refs. [9, 22, 23]. Not surprisingly the singular part of the field, which exerts no force on the particle itself, appears as approximately the Coulomb potential to a local observer moving with the particle.

Our proposal for solving Eq. (11), and determining the self-force acting back on the particle now appears elementary. First we define

$$
\tilde{\psi}^{S} \equiv q / \rho
$$


as a specific approximation to $\psi^{S}$. By construction, we know that $\tilde{\psi}^{S}$ is singular at the particle and is $C^{\infty}$ elsewhere. Also, within a neighborhood of the worldline of the particle

$$
\begin{aligned}
\nabla^{a} \nabla_{a} \tilde{\psi}^{S}= & -4 \pi q \int_{\gamma} \delta^{(4)}(x-z(\tau)) d \tau+O\left(\rho / \mathcal{R}^{4}\right), \\
& \text { as } \rho \rightarrow 0 .
\end{aligned}
$$

Next, we introduce a window function $W$ which is a $C^{\infty}$ scalar field with

$$
W=1+O\left(\rho^{4} / \mathcal{R}^{4}\right) \text { as } \rho \rightarrow 0,
$$

and $W \rightarrow 0$ sufficiently far from the particle, in particular in the wavezone. Finally we define a regular remainder field

$$
\psi^{R} \equiv \psi^{\mathrm{ret}}-W \tilde{\psi}^{S}
$$

which is a solution of

$$
\nabla^{a} \nabla_{a} \psi^{R}=-\nabla^{a} \nabla_{a}\left(W \tilde{\psi}^{S}\right)-4 \pi q \int_{\gamma} \delta^{(4)}(x-z(\tau)) d \tau
$$

from Eq. (11).

The effective source of this equation

$$
S_{\mathrm{eff}} \equiv-\nabla^{a} \nabla_{a}\left(W \tilde{\psi}^{S}\right)-4 \pi q \int_{\gamma} \delta^{(4)}(x-z(\tau)) d \tau
$$

is straightforward to evaluate analytically, and the two terms on the right hand side have delta-function pieces that precisely cancel at the location of the charge, leaving a source which behaves as

$$
S_{\text {eff }}=O\left(\rho / \mathcal{R}^{4}\right) \text { as } \rho \rightarrow 0 .
$$

Thus the effective source $S_{\text {eff }}$ is continuous but not necessarily differentiable, $C^{0}$, at the particle while being $C^{\infty}$ elsewhere 34]. Fig. 1 shows the source function which is actually used in the numerical analysis described in \$IV The modest non-differentiability of $S_{\text {eff }}$ at the particle is revealed in Fig. 2 .

A solution $\psi^{R}$ of

$$
\nabla^{a} \nabla_{a} \psi^{R}=S_{\mathrm{eff}}
$$

is necessarily $C^{2}$ at the particle, and its derivative there provides the self force acting on the particle. Also, in the wavezone $W$ effectively vanishes and $\psi^{R}$ is then identically $\psi^{\text {ret }}$ and provides both the waveform as well as any desired flux measured at a large distance.

General covariance dictates that the behavior of $S_{\text {eff }}$ in Eq. (9) may be analyzed in any coordinate system. But, only in the specific coordinates of Refs. [20] and [21] is it so easily shown [9] that the simple expression for $\psi^{S}$ in Eq. (3) leads to the $O\left(\rho / \mathcal{R}^{4}\right)$ behavior in Eq. (10) and then to the $C^{2}$ nature of the solution $\psi^{R}$ of Eq. (11).

We describe the procedure of solving Eq. (11) as field regularization. Then the derivatives of $\psi^{R}$ determine the self-force, and $\psi^{R}$ is identical to $\psi^{\text {ret }}$ in the wave zone. With this process there is no apparent reason to determine the actual retarded field. However, if one wants to compare results from field regularization with results from a traditional determination of the retarded field then simply adding $W \tilde{\psi}^{S}$ to the remainder $\psi^{R}$ results in the retarded field $\psi^{\text {ret }}$. Such a comparison for our trial of field regularization appears in Figs. (9) and (10).

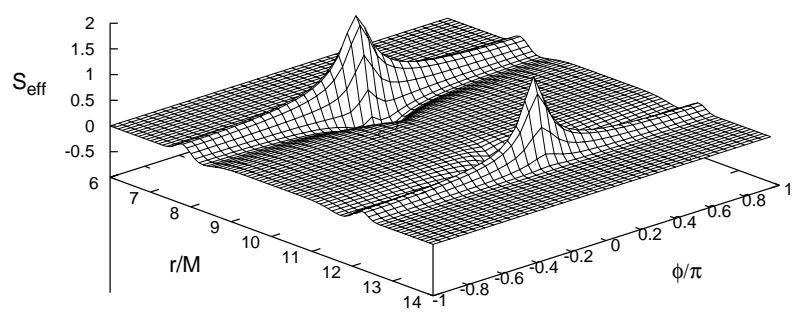

FIG. 1: The effective source $S_{\text {eff }}$ on the equatorial plane. The particle is at $r / M=10, \phi / \pi=0$, where $S_{\text {eff }}$ appears to have no structure on this scale. The smooth "double bump" shape far from the charge is a characteristic of any function similar to $\nabla^{2}\left(W /\left|\vec{r}-\vec{r}_{0}\right|\right)$ in flat space, with a window function $W$ as given in Eq. (18).

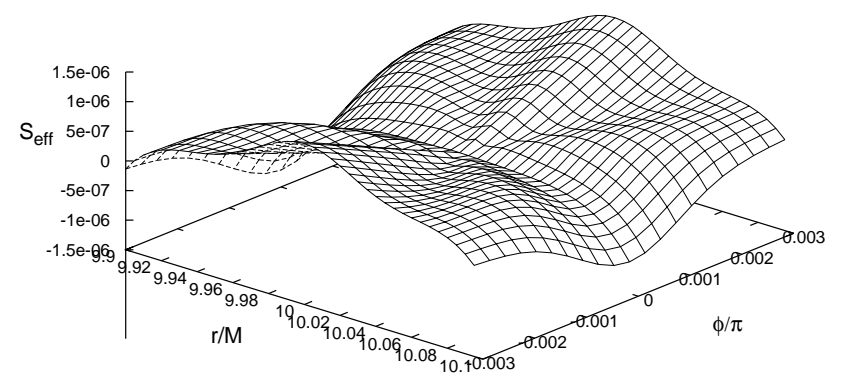

FIG. 2: The effective source $S_{\text {eff }}$ in the equatorial plane in the vicinity of the point source at $r / M=10, \phi / \pi=0$. Note the significant difference of scales with Fig. 1. 


\section{NUMERICAL IMPLEMENTATION}

As a concrete example and test of the field regularization prescription, we apply it to the well-studied case of a scalar charge moving in a circular orbit about a Schwarzschild black hole. We choose $q / m=1$ for the charge to mass ratio of the particle, and a circular geodesic at Schwarzschild radii, $R=10 M$ and $R=12 M$, where $M$ is the mass of the black hole. We work in Schwarzschild coordinates, in which the metric is expressed as $g_{a b}=\operatorname{diag}(-(1-2 M / r), 1 /(1-$ $\left.2 M / r), r^{2}, r^{2} \sin ^{2} \theta\right)$.

For this task, we have developed code that (a) solves the regularized wave equation (11) and (b) computes the scalar self-force. For simplicity, we have chosen to solve the regularized wave equation using a $(1+1)$-approach. We exploit the spherical symmetry of the background, decompose physical quantities into spherical harmonics, and then solve the resulting set of $(1+1) \mathrm{D}$-wave equations (one 'time' + one 'space') for the spherical-harmonic components.

It must be stressed at this point that the numerical implementation presented in this paper does not highlight the advantages of our prescription. The simplicity of the orbit we consider and the spherical symmetry of our background geometry naturally lend themselves to a significantly more efficient frequency-domain approach. But the point here is to provide a quick, first check of our ideas. One is cautioned not to let the simplicity of the present problem obscure the generality of our proposed method, and its potential for cases with generic orbits and spacetimes lacking symmetry, and for self-consistent evolutions which are likely to require self-force calculations in real time (as opposed to being a post-processing step). Testing the robustness of our method against these more difficult problems will be addressed in future work. The current goal is mainly to establish plausibility: to provide both an initial proof-of-principle for the method and also the necessary practice en route to tackling more interesting problems handled using more sophisticated numerical techniques.

\section{A. Scalar fields in a Schwarzschild geometry}

Wave equations in spherically-symmetric backgrounds simplify considerably with a spherical-harmonic decomposition of the field. In the case of a Schwarzschild geometry expressed in Schwarzschild coordinates, this decomposition is typically performed as follows:

$$
\psi=\sum_{l m} \frac{1}{r} f_{l m}(r)(t, r) Y_{l m}(\theta, \phi) .
$$

With $r_{*}=r+2 M \ln (r / 2 M-1)$, this yields equations for $f_{l m}\left(t, r_{*}\right)$ :

$$
-\frac{\partial^{2} f_{l m}}{\partial t^{2}}+\frac{\partial^{2} f_{l m}}{\partial r_{*}^{2}}-V\left(r_{*}\right) f_{l m}=S_{l m}\left(t, r_{*}\right)
$$

where $V\left(r_{*}\right)$ is implicitly given in terms of $r$ as:

$$
V\left(r_{*}\right)=\left(1-\frac{2 M}{r}\right)\left[\frac{l(l+1)}{r^{2}}+\frac{2 M}{r^{3}}\right],
$$

while the source $S_{l m}\left(t, r_{*}\right)$ is

$$
S_{l m}\left(t, r_{*}\right)=(r-2 M) \int \rho\left(x_{\alpha}^{\prime}\right) Y_{l m}\left(\theta^{\prime}, \phi^{\prime}\right) d \Omega^{\prime} .
$$

In a frequency-domain approach, one further chooses to Fourier-decompose $f_{l m}\left(t, r_{*}\right)=\int F_{l m \omega}\left(r_{*}\right) \exp (-i \omega t) d \omega$, and thereby solve the resulting set of ordinary differential equations for $F_{l m \omega}\left(r_{*}\right)$, for each mode $\omega$. This method tends to be numerically expensive, however, for sources with a continuous $\omega$-spectrum. Instead, we choose to solve Eq. (13) as an initial boundary value problem, in a time-domain fashion, for each $(l, m)$. This is done with $S_{l m}$ computed beforehand as the spherical harmonic components of the effective source found in Eq. (9).

\section{B. Effective source term}

A novel feature of our approach is the use of an effective source that permits the easy calculation of both self-forces and fluxes. As discussed above, this effective source is formally

$$
S_{\mathrm{eff}}=-\nabla^{2}\left(W \tilde{\psi}^{S}\right)-4 \pi q \int_{\gamma} \delta^{(4)}(x-z(\tau)) d \tau .
$$

To lowest order, the singular field takes on the form

$$
\psi^{S} \approx \tilde{\psi}^{S}=\frac{q}{\rho} .
$$

We take advantage of the results in [9], where $\rho$ is expressed explicitly as $\rho=\sqrt{\eta_{i j} x^{i} x^{j}}$ in Thorne-HartleZhang coordinates for a particle moving in a circular orbit. Using the coordinate transformation found in Appendix B of [9], where a more detailed discussion of the singular field is found, we are able to express the singular field in Schwarzschild coordinates. (A brief discussion of this coordinate transformation is provided in Appendix A). To complete our effective source, we select a window function whose role is to kill off smoothly the singular field in regions where it is not needed. Consequently, the effective support of the windowed singular field $W \tilde{\psi}^{S}$ is confined to a compact region surrounding the particle's world line.

Our chosen window function is spherically-symmetric with respect to the center of the black hole. This choice was not necessary but guarantees that $W$ would not unnecessarily modify the $(l, m)$-spectrum of the source, and thereby allows us to make more controlled comparisons with existing frequency-domain results on the same problem. Our simple choice of $W$ is

$$
W(r)=\exp \left[-\frac{(r-R)^{N}}{\sigma^{N}}\right] .
$$


In this window function, the constant $\sigma$ sets the width, and the exponent $N$ controls how quickly $W$ and $\nabla_{a} W$ reach the required values of 1 and 0 , respectively, as one approaches the particle. We use $\sigma=2 M$ and $N=8$ in all the results presented in this paper. It is necessary that $N$ is an even integer, and taking full advantage of the accuracy of our approximation for $\psi^{S}$ requires that $W=1+O\left(\rho^{4} / \mathcal{R}^{4}\right)$ as $\rho \rightarrow 0$. Thus we require that $N \geq$ 4. In fact we used $N=8$ in anticipation of improving the approximation for $\psi^{S}$ in the future.

Our choice for the window function leads to the effective source $S_{\text {eff }}$ displayed in Figs. (1) and (2). A larger choice for $\sigma$ would spread the bumps out further, and a smaller choice for $N$ would smooth the bumps. But if $N$ were less than 4 , then $W \tilde{\psi}^{S}$ would not adequately match the behavior of $\psi^{S}$ as $\rho \rightarrow 0$.

With the effective source constructed as above, its spherical-harmonic components were then computed. Circular orbits proved advantageous here because of which the time dependence of the components could then simply be inferred. The spherical harmonic components were evaluated with a 4th-order Runge-Kutta integrator with self-adjusting step size, which was derived from a routine in 24].

\section{Evolution algorithm}

The integration scheme we use in evolving Eq. (13) follows a technique first introduced by Lousto and Price [25], and later improved to fourth-order accuracy by Lousto [26] and Haas [14]. Unlike their schemes, however, we do not deal with sourced and vacuum regions of our numerical domain separately. Their use of a singular delta-function source meant that the resulting field was non-differentiable at the location of the charge, while smooth everywhere else. For us, the effective source is $C^{0}$, implying that the field is at least $C^{2}$. While this is still of finite differentiability, we find that the effective source is differentiable enough not to warrant a treatment different from the vacuum case.

In the $\left(t, r_{*}\right)$-plane, we introduce a staggered grid with step sizes $\Delta t=\frac{1}{2} \Delta r_{*}=h$. In this grid, a unit cell is defined to be the diamond region with corners $\{(t+$ $\left.\left.h, r_{*}\right),\left(t-h, r_{*}\right),\left(t, r_{*}+h\right),\left(t, r_{*}-h\right)\right\}$. Only at these grid points do we evaluate $f_{l m}$. We henceforth drop the spherical-harmonic indices in $f_{l m}$ for convenience.

The main idea behind the algorithm is to integrate the wave equation over a unit cell. This is done easiest with Eddington-Finkelstein null coordinates $u=t-r_{*}$ and $v=t+r_{*}$ as the integration variables.

The differential operator of the wave equation, when expressed in $(u, v)$ coordinates, is just $-4 \partial_{u} \partial_{v}$. Over a unit cell then, the derivative term in Eq. (13) can be

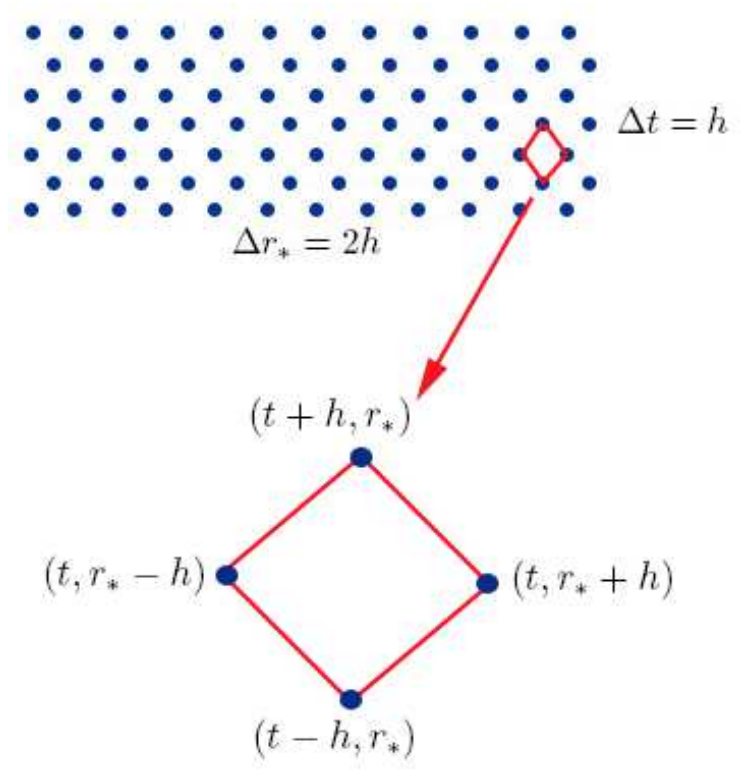

FIG. 3: Staggered (characteristic) grid with unit cell.

integrated exactly:

$$
\begin{aligned}
\iint_{C}-4 \partial_{u} \partial_{v} f d u d v & =-4\left[f\left(t+h, r_{*}\right)+f\left(t-h, r_{*}\right)\right. \\
& \left.-f\left(t, r_{*}+h\right)-f\left(t, r_{*}-h\right)\right] .
\end{aligned}
$$

Integrations of the potential term and the source term do not enjoy the same simplicity as the derivative term. We need to approximate these integrals to the appropriate order in $h$ so as to achieve the desired $O\left(h^{4}\right)$ convergence over the entire numerical domain.

Suppose we wish to solve the wave equation over a region defined by $\Delta T$ and $\Delta R_{*}$. In this region, there will be $N=\Delta T \Delta R_{*} / h^{2}$ cells. Achieving $O\left(h^{4}\right)$-convergence for evolution means that we need to integrate the wave equation with an over-all error of at most $O\left(h^{4}\right)$ over the entire computational domain. For a unit cell, this means an approximation with an error $O\left(h^{4}\right) / N \sim O\left(h^{6}\right)$.

Such an approximation is achieved with the double Simpson rule. Consider a sufficiently differentiable function $G\left(t, r_{*}\right)$ to be integrated over a unit cell. The double Simpson rule then reads:

$$
\begin{array}{rl}
\iint_{C} & G d u d v=\left(\frac{h}{3}\right)^{2}\left[G_{\text {corners }}+16 G\left(t, r_{*}\right)\right. \\
& +4\left(G\left(t+h / 2, r_{*}-h / 2\right)+G\left(t+h / 2, r_{*}+h / 2\right)\right. \\
& \left.\left.+G\left(t-h / 2, r_{*}-h / 2\right)+G\left(t-h / 2, r_{*}+h / 2\right)\right)\right] \\
& +O\left(h^{6}\right),
\end{array}
$$

where $G_{\text {corners }}$ is just the sum of the values of $G$ evaluated at the corners of the unit cell.

This is directly applied in integrating the source term of Eq. (13):

$$
\iint_{C} S_{l m}^{\text {eff }} d u d v
$$


One simply evaluates the source term at the required points and then sums these accordingly in order to get an $O\left(h^{6}\right)$-accurate approximation to the integral.

However, for integrating the potential term:

$$
\iint_{C}-V f d u d v
$$

we recall that one has only restricted access to $f$. The direct evaluation of $f$ is done only at the grid points, i.e. corners of the unit cell. Thus far, only $G_{\text {corners }}$ in Eq. (20) can be explicitly evaluated. To use Eq. (20) for the potential term, we need to determine how to evaluate $f$ at all the other points.

Following Lousto [26], we evaluate $G=-V f$ at the central grid point (i.e. $\left.G\left(t, r_{*}\right)\right)$ using values at the neighboring grid points on the same time slice.

$$
\begin{aligned}
G\left(t, r_{*}\right)= & \frac{1}{16}\left[9 G\left(t, r_{*}-h\right)+9 G\left(t, r_{*}+h\right)\right. \\
& \left.-G\left(t, r_{*}-3 h\right)-G\left(t, r_{*}+3 h\right)\right]+O\left(h^{4}\right) .
\end{aligned}
$$

Note that this is different from Haas 14], who uses grid points in the causal past of the unit cell. The $O\left(h^{4}\right)$-error incurred in this approximation is tolerable because of the $h^{2}$-factor that appears in Eq. (20).

We seek similar approximations for $G$ in the remaining points. Consider first the pair $G\left(t+h / 2, r_{*}-h / 2\right)$ and $G\left(t-h / 2, r_{*}-h / 2\right)$. (The other pair, composed of $G\left(t+h / 2, r_{*}+h / 2\right)$ and $G\left(t-h / 2, r_{*}+h / 2\right)$, is treated similarly). This pair makes up the top and bottom corners of a smaller cell, $\mathcal{C}_{\text {left }}$, made up of the points $\left\{\left(t+h / 2, r_{*}-h / 2\right),\left(t-h / 2, r_{*}-h / 2\right),\left(t, r_{*}-h\right),\left(t, r_{*}\right)\right\}$.

What we shall do next is find an approximation for

$$
G\left(t+h / 2, r_{*}-h / 2\right)+G\left(t-h / 2, r_{*}-h / 2\right)
$$

accurate to $O\left(h^{4}\right)$. Again, this is sufficient because of the $h^{2}$-factor in Eq. (20).

Consider integrating the wave equation over this smaller cell, but this time only up to an accuracy of $O\left(h^{4}\right)$. The integral over the derivative term will again be exact:

$$
\begin{aligned}
\iint_{C_{\text {left }}}-4 \partial_{u} \partial_{v} f d u d v & =-4\left[f\left(t+h / 2, r_{*}-h / 2\right)\right. \\
& \left.+f\left(t-h / 2, r_{*}-h / 2\right)-f\left(t, r_{*}-h\right)-f\left(t, r_{*}\right)\right] .
\end{aligned}
$$

The integrals of the potential and source terms over this smaller cell are again handled as before, but this time we approximate them only to $O\left(h^{4}\right)$. To this end, the double trapezoidal rule will suffice, which reads:

$$
\begin{aligned}
& \iint_{C_{\text {left }}} G d u d v=\left(\frac{h}{2}\right)^{2}\left[G\left(t+h / 2, r_{*}-h / 2\right)\right. \\
& \left.\quad+G\left(t-h / 2, r_{*}-h / 2\right)+G\left(t, r_{*}-h\right)+G\left(t, r_{*}\right)\right] \\
& \quad+O\left(h^{4}\right) .
\end{aligned}
$$

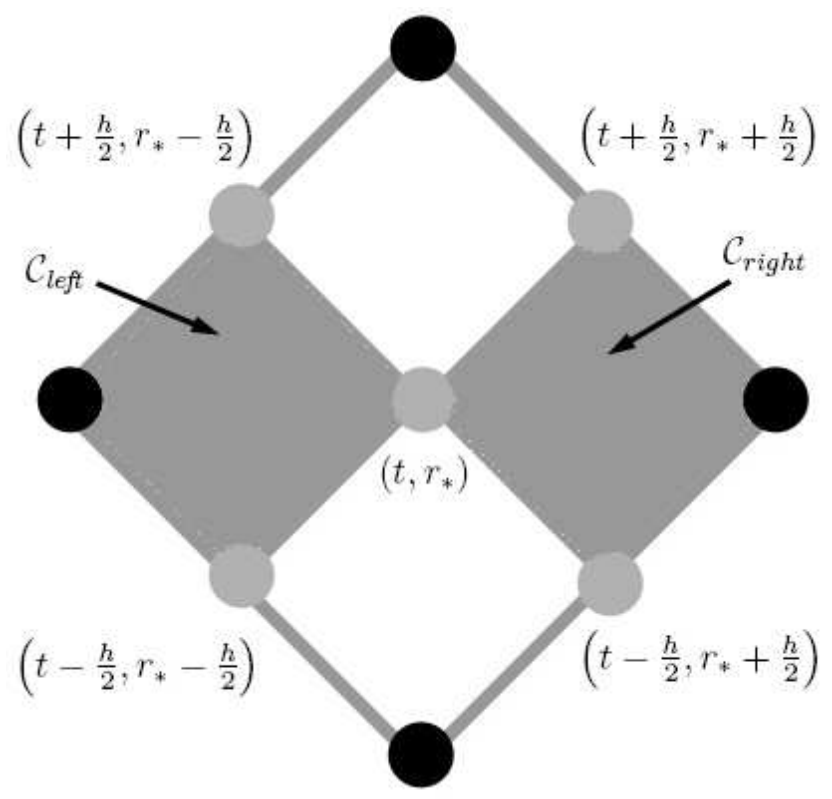

FIG. 4: Unit cell of the algorithm. The black dots indicate grid points, whereas the gray ones stand for the points where $G=-V f$ needs to be approximated. The subcells $\mathcal{C}_{\text {left }}$ and $\mathcal{C}_{\text {right }}$ are shaded gray. To approximate $G$ at some of the gray dots, we integrate the wave equation in each of these subcells.

Applying this to the potential term then gives:

$$
\begin{aligned}
\iint_{C_{\text {left }}}-V f d u d v= & -\left(\frac{h}{2}\right)^{2} \times \\
& {\left[V\left(r_{*}-h / 2\right) f\left(t+h / 2, r_{*}-h / 2\right)\right.} \\
& +V\left(r_{*}-h / 2\right) f\left(t-h / 2, r_{*}-h / 2\right) \\
& +V\left(r_{*}-h\right) f\left(t, r_{*}-h\right) \\
& \left.+V\left(r_{*}\right) f\left(t, r_{*}\right)\right]+O\left(h^{4}\right) .
\end{aligned}
$$

Combining Eq. (25) and Eq. (27), the result of integrating the wave equation over this smaller cell yields:

$$
\begin{aligned}
& f\left(t+h / 2, r_{*}-h / 2\right)+f\left(t-h / 2, r_{*}-h / 2\right)= \\
& \quad\left(f\left(t, r_{*}-h\right)+f\left(t, r_{*}\right)\right)\left[1-\frac{1}{2}\left(\frac{h}{2}\right)^{2} V\left(r_{*}-h / 2\right)\right] \\
& \quad-\frac{1}{4} \iint_{C_{\text {left }}} S_{\text {eff }} d u d v+O\left(h^{4}\right) .
\end{aligned}
$$

After multiplying both sides of this last equation by $-V\left(r_{*}-h / 2\right)$, the resulting left-hand-side becomes two of the as yet missing pieces in the double Simpson formula:

$$
\begin{aligned}
G\left(t+h / 2, r_{*}\right. & -h / 2)+G\left(t-h / 2, r_{*}-h / 2\right)= \\
& -V\left(r_{*}-h / 2\right) f\left(t+h / 2, r_{*}-h / 2\right) \\
& -V\left(r_{*}-h / 2\right) f\left(t-h / 2, r_{*}-h / 2\right) .
\end{aligned}
$$

The resulting equation then gives us the desired $O\left(h^{4}\right)$ approximation of the missing expression, $G\left(t+h / 2, r_{*}-\right.$ $h / 2)+G\left(t-h / 2, r_{*}-h / 2\right)$, in Eq. (20). Following the 
same steps, it is easy to arrive at an equivalent approximation for the other missing pair, $G\left(t+h / 2, r_{*}+h / 2\right)+$ $G\left(t-h / 2, r_{*}+h / 2\right)$. We summarize these below:

$$
\begin{aligned}
G(t+h / 2, & \left.r_{*}-h / 2\right)+G\left(t-h / 2, r_{*}-h / 2\right)= \\
& -V\left(r_{*}-h / 2\right)\left(f\left(t, r_{*}-h\right)+f\left(t, r_{*}\right)\right) \\
& \times\left[1-\frac{1}{2}\left(\frac{h}{2}\right)^{2} V\left(r_{*}-h / 2\right)\right] \\
+ & \frac{V\left(r_{*}-h / 2\right)}{4} \iint_{C_{\text {left }}} S_{\text {eff }} d u d v+O\left(h^{4}\right) .
\end{aligned}
$$

$$
\begin{aligned}
G(t+h / 2, & \left.r_{*}+h / 2\right)+G\left(t-h / 2, r_{*}+h / 2\right)= \\
- & V\left(r_{*}+h / 2\right)\left(f\left(t, r_{*}+h\right)+f\left(t, r_{*}\right)\right) \\
& \times\left[1-\frac{1}{2}\left(\frac{h}{2}\right)^{2} V\left(r_{*}-h / 2\right)\right] \\
+ & \frac{V\left(r_{*}+h / 2\right)}{4} \iint_{C_{\text {right }}} S_{\text {eff }} d u d v+O\left(h^{4}\right) .
\end{aligned}
$$

Except for the presence of integrated source terms, these equations are identical to Lousto's equations (32) and (33), and Haas's equations (2.8) and (2.9).

Following Haas [14], we choose to avoid isolated occurrences of $f\left(t, r_{*}\right)$, which prove to be numerically unstable close to the event horizon. As pointed out in [14], this is due to having first approximated $G=-V f$, which makes it difficult to isolate $f=-G / V$ where $V \approx 0$. This appears unnecessary if $f$ were directly approximated instead of $G$ in (23). Nevertheless, like Haas, we avoid needing to isolate $f$ by adding up equations Eq. (30) and Eq. (31), and then Taylor-expanding the potential terms that are multiplied by $f\left(t, r_{*}\right)$. The result is Haas's equation (2.10) with extra source terms:

$$
\begin{aligned}
\sum G \equiv G(t+h / 2, & \left.r_{*}-h / 2\right)+G\left(t-h / 2, r_{*}-h / 2\right)+G\left(t+h / 2, r_{*}+h / 2\right)+G\left(t-h / 2, r_{*}+h / 2\right)= \\
& -2 V\left(r_{*}\right) f\left(t, r_{*}\right)\left[1-\frac{1}{2}\left(\frac{h}{2}\right)^{2}\right]-V\left(r_{*}-h / 2\right) f\left(t, r_{*}-h\right)\left[1-\frac{1}{2}\left(\frac{h}{2}\right)^{2} V\left(r_{*}-h / 2\right)\right] \\
& -V\left(r_{*}+h / 2\right) f\left(t, r_{*}+h\right)\left[1-\frac{1}{2}\left(\frac{h}{2}\right)^{2} V\left(r_{*}+h / 2\right)\right] \\
& -\frac{1}{2}\left[V\left(r_{*}-h / 2\right)-2 V\left(r_{*}\right)+V\left(r_{*}+h / 2\right)\right]\left(f\left(t, r_{*}-h\right)+f\left(t, r_{*}+h\right)\right) \\
& +\frac{V\left(r_{*}-h / 2\right)}{4} \iint_{C_{\text {left }}} S_{\text {eff }} d u d v+\frac{V\left(r_{*}+h / 2\right)}{4} \iint_{C_{\text {right }}} S_{\text {eff }} d u d v+O\left(h^{4}\right)
\end{aligned}
$$

This last equation completes the pieces needed for the evolution algorithm.

Using Eq. (19) for the derivative term and Eq. (20) for the potential and source terms, the result of integrating the wave equation over the unit cell finally yields:

$$
\begin{aligned}
f\left(t+h, r_{*}\right)= & -f\left(t-h, r_{*}\right)+\frac{\left[1-\frac{1}{4}\left(\frac{h}{3}\right)^{2} V\left(r_{*}+h\right)\right]}{\left[1+\frac{1}{4}\left(\frac{h}{3}\right)^{2} V\left(r_{*}\right)\right]} f\left(t, r_{*}+h\right)+\frac{\left[1-\frac{1}{4}\left(\frac{h}{3}\right)^{2} V\left(r_{*}-h\right)\right]}{\left[1+\frac{1}{4}\left(\frac{h}{3}\right)^{2} V\left(r_{*}\right)\right]} f\left(t, r_{*}-h\right) \\
& -\frac{1}{\left[1+\frac{1}{4}\left(\frac{h}{3}\right)^{2} V\left(r_{*}\right)\right]}\left[\left(\frac{h}{3}\right)^{2}\left(4 G_{0}+\sum G\right)+\frac{1}{4} \iint_{C} S_{\text {eff }} d u d v\right]+O\left(h^{6}\right),
\end{aligned}
$$

where $G_{0}$ is evaluated according to Eq. (23), with $G\left(t, r_{*}\right)=-V\left(r_{*}\right) f\left(t, r_{*}\right) ; \sum G$ is the expression in Eq. (32); and the double Simpson rule Eq. (20) is applied in evaluating the remaining integral term $\iint_{C} S_{\text {eff }} d u d v$. With this equation, one can now determine the field $f$ at time $t+h$ given its values at earlier times $t$ and $t-h$.

This derivation makes liberal use of double Simpson and double trapezoidal formulas when approximating integrals of the source and potential terms over the unit cell. The formulas come from their single-integral coun- terparts:

$$
\int_{x_{0}}^{x_{0}+h} f(x) d x=\frac{h}{2}\left[f\left(x_{0}\right)+f\left(x_{0}+h\right)\right]-\frac{h^{3}}{12} f^{(2)}(\xi)
$$

$$
\begin{gathered}
\int_{x_{0}}^{x_{0}+2 h} f(x) d x=\frac{h}{3}[ \\
{\left[\left(x_{0}\right)+4 f\left(x_{0}+h\right)+f\left(x_{0}+2 h\right)\right]} \\
-\frac{h^{5}}{90} f^{(4)}(\xi)
\end{gathered}
$$


where $f^{(n)}$ denotes the $n$ th-derivative of $f$, and $\xi$ is some point within the limits of integration. These require the boundedness, if not existence of the second and fourth derivatives of the integrand for the error estimate to be valid. With the limited differentiability of our source and potential terms $\left(C^{0}\right.$ and $C^{2}$, respectively), one might worry about the validity of our over-all convergence estimate. However, our calculations reveal that 4th-order convergence is achieved despite this deficiency.

\section{Initial data and boundary conditions}

For our evolution we have no obvious method for choosing a priori the correct initial data, which consists of the value of $\psi^{R}$ on two consecutive constant-time slices. Consequently we just set the initial $\psi^{R}$ to zero everywhere on the initial two slices. Physically, this scenario corresponds to the impulsive appearance of the scalar point charge along with $W \tilde{\Psi}^{S}$, which leads to spurious radiation contaminating our computational domain during the early stages of the evolution. Fortunately, this radiation propagates out of the regions of interest quickly; so to circumvent the need for proper initial data, we simply evolve the equation to long enough times such that initial data effects do not become pertinent in any of our results.

With the scalar charge moving in a circular orbit, it is expected that the field eventually becomes stationary in a frame corotating with the charge. A practical test then for the persistence of initial data effects is to simply check whether or not the field has already settled into a quiescent state when evaluated in this frame.

Boundary conditions are treated similarly. Rather than handling them carefully, we instead made the computational domain large enough that errors incurred by unspecified boundary conditions did not affect our regions of interest. For this work, our choice of boundaries were at $r_{*}=-700 M$ and $r_{*}=800 M$.

\section{E. Self-force calculation}

At the end of evolution for each mode, we compute the self-force at the location of the particle. Since this location is not on any grid point, interpolation of $f_{l m}(T, r)$ and its derivatives to $r=R$ was required using a selection of grid points surrounding it.

Once this was done, computing the self-force was a simple matter of performing the following sums:

$$
\begin{aligned}
\psi^{R}= & \frac{1}{R} \sum_{l=0}^{L} f_{00}(T, R) Y_{00}\left(\frac{\pi}{2}, \Omega T\right) \\
& +\frac{2}{R} \sum_{l=1}^{L} \sum_{m=1}^{l} \operatorname{Re}\left(f_{l m}(T, R) Y_{l m}\left(\frac{\pi}{2}, \Omega T\right)\right)
\end{aligned}
$$

$$
\begin{aligned}
\partial_{t} \psi^{R}= & \frac{2}{R} \sum_{l=0}^{L} \sum_{m=1}^{l}(m \Omega) \operatorname{Im}\left(f_{l m}(T, R) Y_{l m}\left(\frac{\pi}{2}, \Omega T\right)\right) \\
\partial_{r} \psi^{R}= & \frac{1}{R} \sum_{l=0}^{L} \partial_{r} f_{00}(T, R) Y_{00}\left(\frac{\pi}{2}, \Omega T\right) \\
& +\frac{2}{R} \sum_{l=1}^{L} \sum_{m=1}^{l} \operatorname{Re}\left(\partial_{r} f_{l m}(T, R) Y_{l m}\left(\frac{\pi}{2}, \Omega T\right)\right)
\end{aligned}
$$

Here, $L$ is the point where we truncate the multipole expansion. In all our work we have used $L=39$. These sums arise primarily because our charge moves in a circular orbit.

Two methods were employed for interpolation. The first was a simple Lagrange interpolation of both $f$ and $\partial_{r} f$ to $r=R$. However, because of the finite differentiability of our regular field at $r=R$ we also interpolated using the form

$$
\psi^{R}(r)=A_{0}+A_{1} x+A_{2} x^{2}+A_{3} x^{3}+\theta(x) B_{0} x^{3},
$$

where $x=r-R$, and $\theta(x)$ is the standard Heaviside function. This form closely respects the $C^{2}$ nature of the regular field at $r=R$ by allowing for a discontinuity in the third derivative.

With this form, $\left.\left(\nabla_{r} F\right)\right|_{r=R}=A_{1}$. However, this led to results not significantly different from the one achieved with ordinary Lagrange interpolation.

\section{CODE DIAGNOSTICS}

\section{A. Convergence}

The convergence of a time-domain code is easily determined by computing the convergence factor $n$ as defined by Lousto 26]:

$$
\begin{aligned}
n\left(r_{*}, t\right)= & \log \left|\frac{f_{4 h}\left(r_{*}, t\right)-f_{2 h}\left(r_{*}, t\right)}{f_{2 h}\left(r_{*}, t\right)-f_{h}\left(r_{*}, t\right)}\right| / \log (2) \\
& +\log \left|\epsilon^{(n)}(\xi)\right| / \log (2),
\end{aligned}
$$

where $f_{\Delta}\left(r_{*}, t\right)$ is the result of the evolution for a resolution of $\Delta$, and $\epsilon^{(n)}(\xi)$ represents an error function $\approx 1$. An $n$ th-order evolution code is one for which $\psi=\psi_{N}(h)+\left(\epsilon^{(n)}\right)(\xi) h^{n}$, where $\psi_{N}(h)$ is the numerical solution at resolution $h$.

In checking convergence, one evolves the wave equation at different resolutions, $h, 2 h$, and $4 h$. For a fixed $r_{*}=R$, one then extracts $f_{h}(R, t), f_{2 h}(R, t)$, and $f_{4 h}(R, t)$ for all $t$. From these, one can compute $n(R, t)$.

The convergence factor was computed for a few representative points in the wavezone and in the region close to the point particle. Two of these are shown in Fig. [5. 
These are for $r \approx 10 M$ and $r \approx 100 M$. All show the desired 4th-order convergence eventually, following a transient period in which the numerical evolution is contaminated by the effects of poor initial data.

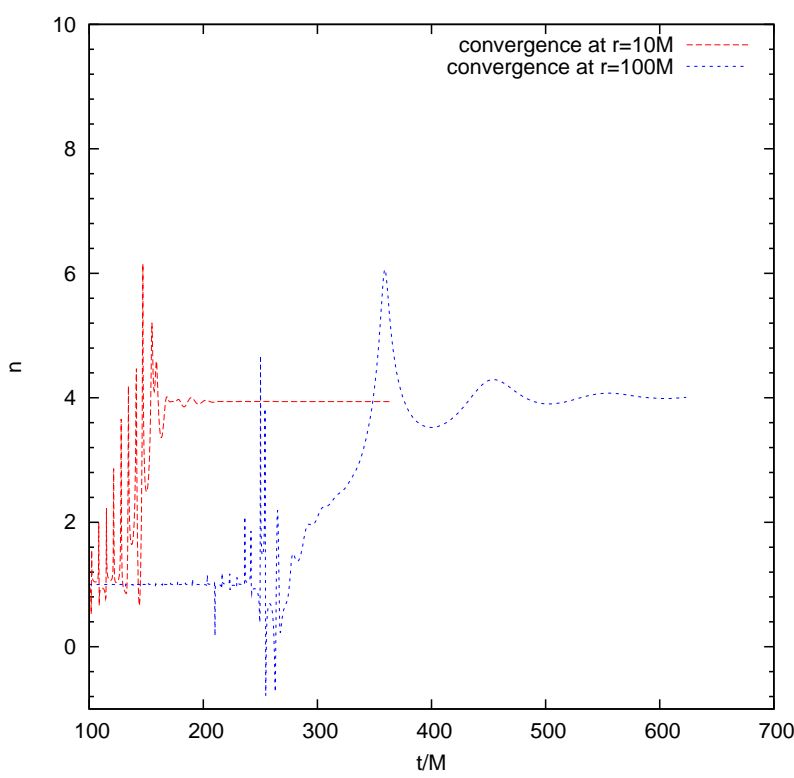

FIG. 5: Convergence at the particle location $(r=10 M)$ and in the wavezone $(r=100 M)$. At the start of the evolution, inequivalent initial data lead to the lack of 4 th-order convergence. But $n$ gradually approaches 4 as initial-data effects propagate away from the computational domain. Note that the convergence test at the particle location already includes the interpolation step.

\section{B. High- $l$ fall-off}

In [4, 5, 7, 9], it was demonstrated that the rate of convergence of the $l$-components of the self-force was dictated primarily by the lack of differentiability of the regular piece from which the self-force is computed. By definition, the difference between the retarded field and the singular field yields a function that is $C^{\infty}$. In this ideal situation, convergence in $l$ of the self-force computed from this smooth regular field would be exponentially fast. In practice, however, one is always limited to constructing only an approximate singular field, therefore leaving nondifferentiable pieces in the residual $\psi^{\text {ret }}-\tilde{\psi}^{S}$. The degree of non-differentiability of this remainder is what sets the rate of convergence of the self-force in $l$.

The high- $l$ asymptotic structure of the singular piece $\psi^{S}$ is such that:

$$
\begin{gathered}
\lim _{r \rightarrow R}\left(\nabla_{r} \psi^{S}\right)_{l}=\left(l+\frac{1}{2}\right) A_{r}+B_{r}-\frac{2 \sqrt{2} D_{r}}{(2 l-1)(2 l+3)} \\
+\frac{E_{r}^{(1)} \mathcal{P}_{3 / 2}}{(2 l-3)(2 l-1)(2 l+3)(2 l+5)}+\ldots,
\end{gathered}
$$

where $A, B, D, \ldots$ are the regularization parameters, which commonly appear in contemporary self-force studies $[4,5]$.

The number of regularization parameters that can be determined in this expansion corresponds directly to the accuracy of the singular field approximation. Convergence in $l$ of the self-force $\nabla_{r} \psi^{R}$ is then fixed by the lowest-order undetermined piece of the approximate singular field. Specifically, if the singular field is accurately determined only up to the $B$-term of the expansion above, then the $l$-convergence of the self-force would be $\sim 1 / l^{2}$, corresponding to the $D$-term fall-off.

The approximation to the singular field here is $\tilde{\psi}^{S}=$ $q / \rho$, and the attendant THZ-Schwarzschild coordinate transformation, has been shown in [9] to include at least the $D$-term. The expectation then would be for the $l$ components of our remainder, $\left(\nabla_{r} \psi^{R}\right)_{l}$, to fall off as the $E^{(1)}$-piece:

$$
\frac{E_{r}^{(1)} \mathcal{P}_{3 / 2}}{(2 l-3)(2 l-1)(2 l+3)(2 l+5)} .
$$

Fig. 6 shows our results confirming this expectation. Our results are plotted with $D, E^{(1)}$ and $E^{(2)}$ fall-off curves found in Eq. (41) that are made to match at $l=15$.

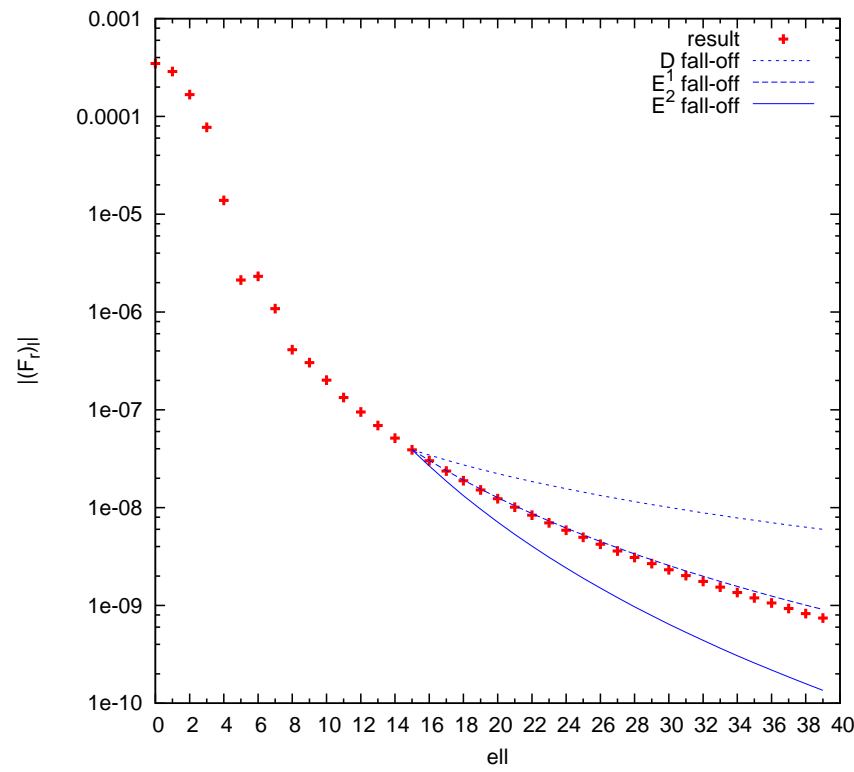

FIG. 6: $\left(\nabla_{r} \psi^{R}\right)_{l}$ versus $l$. Our results show $l$-convergence closest to the $E^{(1)}$ fall-off. The blue lines correspond to the expected fall-off in the $r$-component of the self-force when one regularizes using a singular field approximation without the $D$-term, $E^{(1)}$-term, and $E^{(2)}$-term, respectively. Our result is matched to these curves at $l=15$.

The $t$-component of the self-force, on the other hand, does not require regularization for the case of a charge in a circular orbit of Schwarzschild. An exponential fall-off is then expected. This is shown in Fig. 7. 


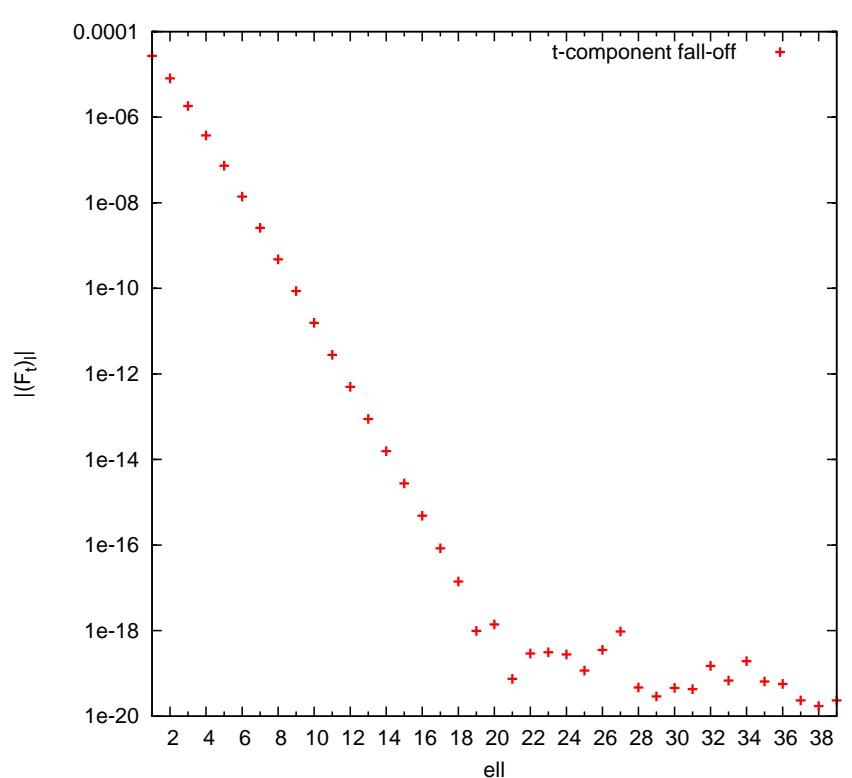

FIG. 7: $\left(\nabla_{t} \psi^{R}\right)_{l}$ versus $l$. The expected exponential fall-off is observed until the point where numerical noise begins to dominate.

\section{Dependence on the window function}

The use of a window function $W$ is a peculiar feature of our approach. Its function is mainly to kill off $\tilde{\psi}^{S}$ in the regions where it is no longer relevant and thereby to have the computed regular field $\psi^{R}$ transform into the retarded field in those regions. As it is a mere artifact of our implementation, it is crucial that the self-force and waveform be independent of the specific choice of window function.

One has considerable freedom in choosing $W$, the only requirements being that $W$ goes to 1 and that its gradient vanishes fast enough in the limit that one approaches the point particle. With our specific choice of $\mathrm{W}$ becoming numerically significant only in an annular region $|r-R| \lesssim$ $\sigma$, we have inspected the changes in the self-force and fluxes as one varies the width $\sigma$.

Fig. 8 shows the effect of doubling the annular support of the window function. The $(l=2, m=2)$ wave equation was evolved for the same length of time, but with effective sources having different window functions. A comparison is then made of the resulting fields over most of the computational domain. It is seen that the fields differ significantly only in regions where the window functions differ. Nevertheless, the regular field $\psi^{R}$ remains the same (up to fractional changes of $\sim 10^{-8}$ in the most physically-relevant regions: the vicinity of the charge, $r=10 M$ (where the self-force is computed), and the wavezone, $r \gg 10 M$ (where the waveform is to be extracted).

As desired then, the window function appears to have no effect on any of the numerical results attained.

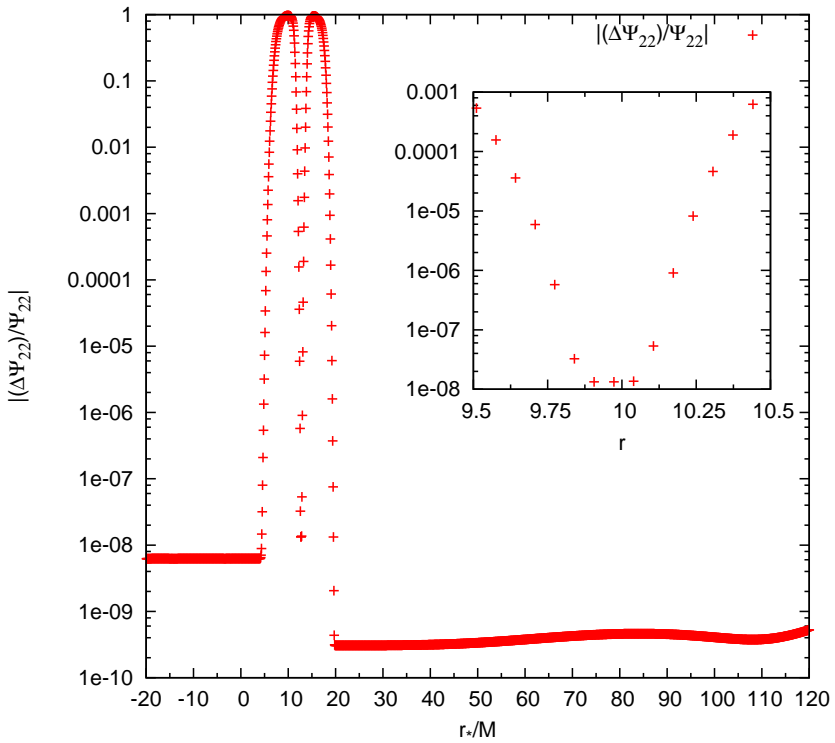

FIG. 8: Fractional changes in $\psi_{22}^{R}$ as a result of using different window functions. Note that these changes are significant only where the window functions differ; they are insignificant in the important regions in the vicinity of the charge, $r=$ $10 M$, and in the wave zone, $r \gg 10 M$.

\section{RESULTS}

\section{A. Recovering the retarded field}

From our numerical calculations we are able to accurately recover the retarded field. In the wavezone, where the singular field is negligible, this retarded field equals our regular field $\psi^{R}$. Since, energy fluxes depend directly on the retarded field in this region, the accuracy with which we recover the retarded field in the wavezone gives us a measure of how well we can compute fluxes using our method. We determine this accuracy by comparing our result for $\psi^{R}$ in the wavezone with that obtained for the retarded field using a separate frequency-domain calculation. An example of such a comparison is shown in Figs.9 and 10. We observe relative errors that are at worst $10^{-6}$. Shown in Fig. 9 are the $(l=2, m=2)$ component of the retarded field computed in the frequency-domain and our corresponding time-domain result, $\psi_{22}^{R}+\left(W \tilde{\psi}^{S}\right)_{22}$, for the case of a charge at $r=10 \mathrm{M}$. Also shown are the singular field $\left(W \tilde{\psi}^{S}\right)_{22}$ and the regular field $\psi_{22}^{R}$.

\section{B. Self-force}

We obtain the $t$ and $r$ components of the self-force for an orbit at radii $R=10 M$ and $12 M$. These are summarized in Table 【.

Fig. [11] shows the convergence of our calculation of the the $(l=2, m=2)$ time component of the self-force we show the convergence of our time-domain calculation to 


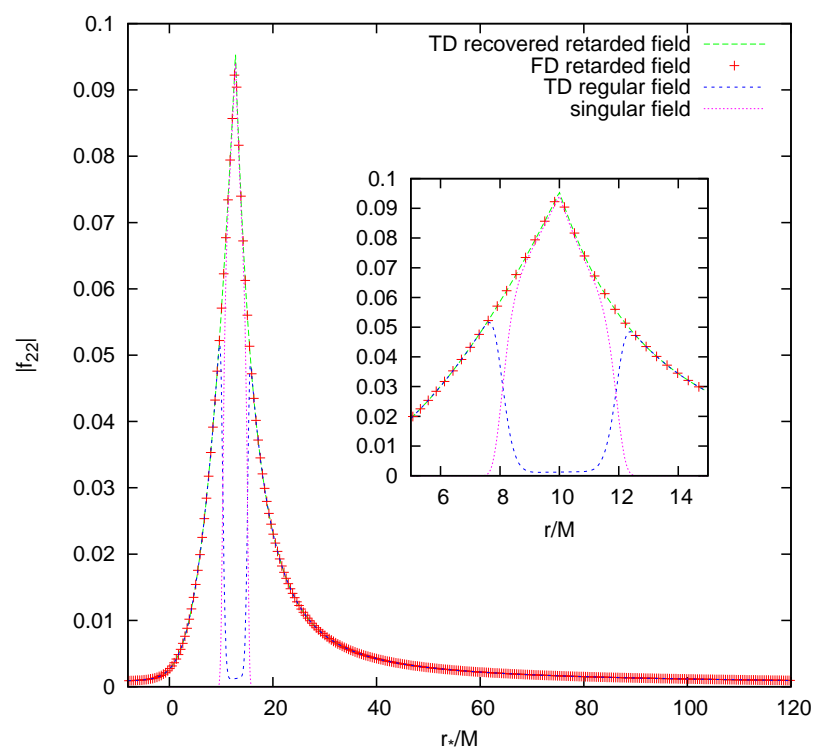

FIG. 9: Comparison of time-domain and frequency-domain results for $f_{22}\left(r_{*}\right)$. The regular field is the result of our code (represented by the blue dashed line). Adding this to the $(l=2, m=2)$-component of our analytical singular field, $W \tilde{\psi}^{S}$, results in the FD-computed retarded field to good agreement.

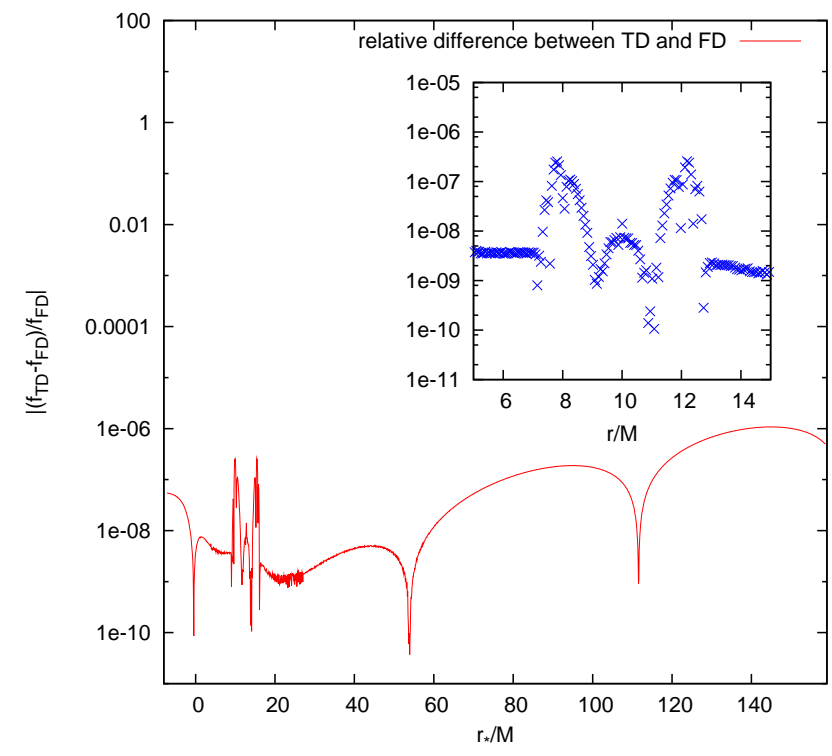

FIG. 10: Relative error between time-domain and frequencydomain results for $f_{22}\left(r_{*}\right)$. Excellent agreement is achieved; errors are at worst $\sim 10^{-6}$.

the frequency-domain result. We have excellent convergence after a time of $200 M$, which is approximately one orbital period.

\begin{tabular}{ccccc}
\hline \hline & $R$ & Time-domain & Frequency-domain & error \\
\hline$\partial_{t} \psi^{R}$ & $10 M$ & $3.750211 \times 10^{-5}$ & $3.750227 \times 10^{-5}$ & $0.000431 \%$ \\
$\partial_{r} \psi^{R}$ & $10 M$ & $1.380612 \times 10^{-5}$ & $1.378448 \times 10^{-5}$ & $0.157 \%$ \\
\hline$\partial_{t} \psi^{R}$ & $12 M$ & $1.747278 \times 10^{-5}$ & $1.747254 \times 10^{-5}$ & $0.00139 \%$ \\
$\partial_{r} \psi^{R}$ & $12 M$ & $5.715982 \times 10^{-6}$ & $5.710205 \times 10^{-6}$ & $0.101 \%$ \\
\hline \hline
\end{tabular}

TABLE I: Summary of self-force results for $R=10 M$ and $R=12 M$. The error is determined by a comparison with an accurate frequency-domain calculation [9] .

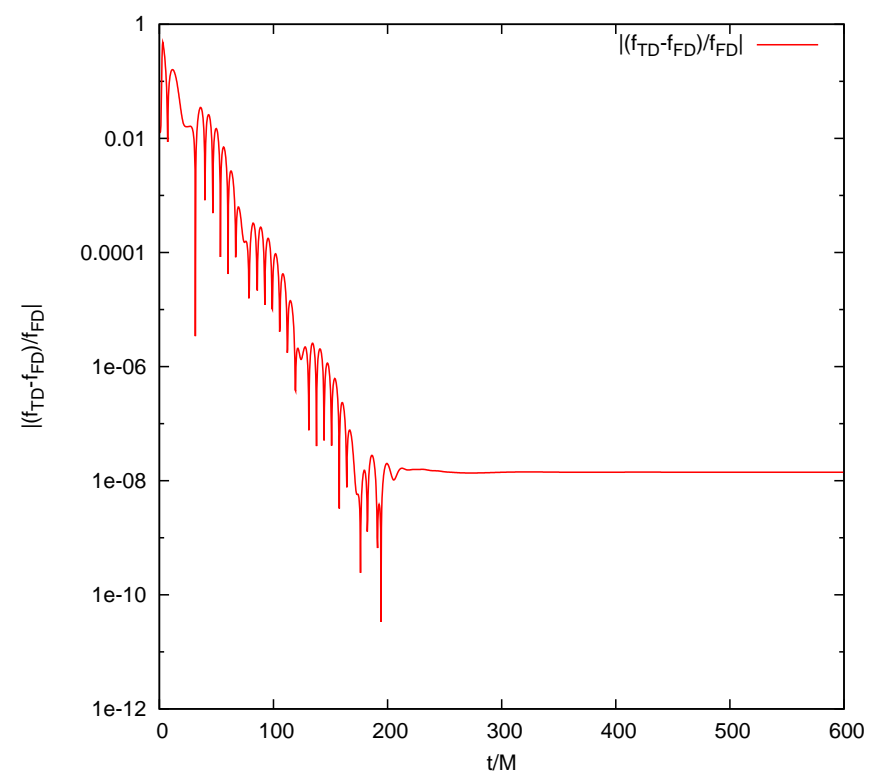

FIG. 11: Relative error in the time-domain calculation of $f_{22}$, as compared with the frequency-domain calculation, versus time at $r$ close to the charge at $10 \mathrm{M}$.

\section{DISCUSSION}

In the specific context of a point charge orbiting a back hole, we have introduced a very general approach suitable for the time-domain generation of waveforms and also the calculation of the backreacting self-force.

Our initial tests are admittedly on the very restrictive case of circular orbits of the Schwarzschild geometry, where we have taken advantage of the spherical symmetry to decompose the source and field into spherical harmonics. This has allowed us to compare our results to available frequency-domain results of very high precision [9, 12]. In some manner, because of our use of a sphericalharmonic decomposition, our analysis might be likened to using spectral methods. But, the method of field regularization inherently does not require a mode-decomposition and could be implemented with a full $(3+1)$ numerical code. For our test case we achieve an extremely accurate calculation for the time component of the self-force $\partial_{t} \tilde{\psi}^{R}$, which is equivalent to the rate of energy lost by radiation. Notably, in our $(1+1)$ implementation, the initial data settled down to provide this accurate component of the self-force within only one orbit of the particle as 
shown in Fig. 11. This might be contrasted with a calculation of $d E / d t$ made from a flux integral evaluated in the wave-zone, which, with similarly unspecified initial data, requires evolution over a substantial number of orbits.

For a circular orbit, the radial component of the selfforce $\partial_{r} \tilde{\psi}^{R}$ is conservative and generally more difficult to calculate. We were able to match more accurate analyses [9, 12, 13, 14] to about $0.1 \%$. With the spherical harmonic decomposition, our analysis went up to $L=39$. This relatively high number is due primarily to the slow polynomial convergence resulting from the mode-decomposition of the self-force. We expect this to be endemic in all self-force calculations that rely on some kind of spectral decomposition, as it is the penalty incurred when one represents objects of limited differentiability in terms of smooth functions.

A technique similar to that described in Ref. [9] could possibly mitigate this weakness. For our specific implementation, we could choose to calculate and sum modes only up to, say $l=15$, and then take advantage of the known asymptotic fall-off in $l$ shown in Eq. (41). Using the computed modes, we determine the coefficients in the expected fall-off for the self-force, and then using these, analytically complete the sum to $l=\infty$. This results in a slightly more accurate result for $\partial_{r} \tilde{\psi}^{R}$. A similar procedure of "fitting" to a known asymptotic fall-off might prove useful if one chooses to implement field regularization using spectral methods.

We expect field regularization to be best implemented on a $(3+1)$ finite-difference code, with mesh refinement in the vicinity of the charge to better resolve the limited differentiability of our analytically constructed source function. Such a process will ameliorate the problem of slow polynomial convergence ailing typical mode-sum prescriptions.

For the EMRI problem today, there is great interest in calculating the rate of energy being radiated for a point mass orbiting a rotating black hole and in using the result to modify the the orbit of the mass with some version of an adiabatic approximation. For a general orbit, the energy flux is not easy to determine. Current methods use the axial symmetry of the Kerr geometry to separate out one dimension, and then deal with a $(2+1) \mathrm{D}$ problem for the radiation from a point mass. The representation of a point mass on a grid is typically problematical. Replacing a $\delta$-function source by a narrow Gaussian [27, 28] is reasonable but does not accurately reproduce frequencydomain results. The recent distribution of a $\delta$-function over a modest number of grid points by Sundararajan et al 29] appears more robust. The strategy laid out here provides a natural remedy to this issue. Instead of dealing with a wave equation with a $\delta$-function source, we solve an equivalent problem with a regular and distributed source. The results displayed in Figures 9 and 10 clearly point to the effectiveness and accuracy of our method.

Beyond the numerical modeling of $\delta$-function sources though, the method of field regularization provides di- rect access to the self-force, which is essential in a fullyconsistent treatment of particle motion and wave generation. Current methods under development are based upon energy and angular momentum flux calculations that will certainly miss conservative self-force effects. These methods rely upon flux integrals evaluated in the wave-zone and some orbit averaging or post-processing to effect the change in orbital energy or angular momentum, which are difficult to implement carefully [30, 31] and to justify rigorously. The more direct approach of locally calculating the self-force to update the particle orbit has been largely avoided because of the prohibitive computational expense associated with mode-sum calculations of the self-force. In a $(3+1)$ finite-differencing implementation of field regularization, calculating the self-force is no more expensive than performing a numerical derivative and possibly an interpolation. As such, it represents a step forward towards the goal of efficiently producing consistent numerical models of particle motion and radiation in curved spacetime.

The recent proposal of Barack, Golbourn and Sago [15, 32, 33] is closest in spirit to our method of field regularization. They model a point charge with a distributed effective source derived instead from their "puncture function", which is quite similar to our $\tilde{\psi}^{S}$. They base their construction of the puncture function on the 'direct'+'tail' decomposition, rather than on the Green function decomposition in [19] that naturally provides our regularizing singular field $\tilde{\psi}^{S}$. Their current puncture function, however, appears to prevent them from calculating a self-force. Moreover, in anticipation of a Kerr background application, they envisage using a $(2+1)$ code, necessitating a mode-sum over a mode index $m$, which will again feature the characteristic polynomial convergence of this approach to self-force calculation. This is demonstrated in Fig. 12. Using our results, we perform partial sums over $l$, i.e.

$$
\left(\nabla_{r} \psi^{R}\right)_{m} \equiv \sum_{l>|m|}^{L}\left(\nabla_{r} \psi^{R}\right)_{l m}
$$

to get the resulting fall-off in $m$. We observe a fall-off close to $1 / \mathrm{m}^{4}$ in the modes. Consequently, if we were to follow Barack et al's $m$-mode prescription, the self-force would converge as $1 / \mathrm{m}^{3}$.

In principle, our method of field regularization appears to resolve two important issues in the context of EMRI simulations: (a) numerically representing $\delta$ function sources, and (b) calculating the self-force. At this time our test of a scalar charge in a circular orbit of the Schwarzschild geometry is a carefully controlled numerical experiment and provides us with detailed information about the relationship between the approximation for $\psi^{S}$ and the rate of convergence of the selfforce. However, our test is also extremely elementary when compared to the actual case of a point mass emitting gravitational waves from a generic orbit of the Kerr geometry, which is most relevant for EMRIs. Future work 


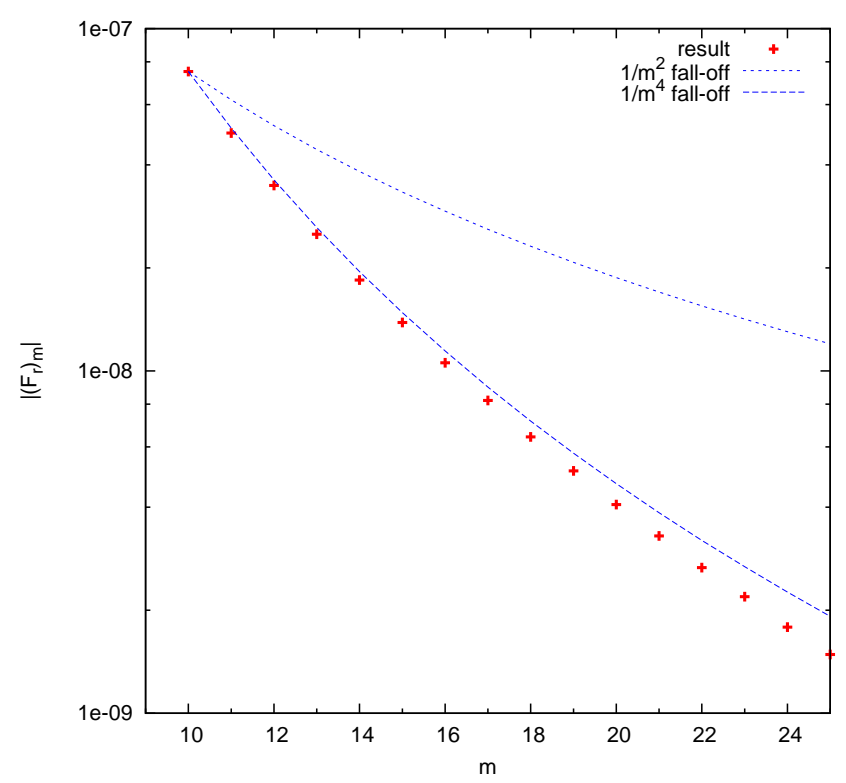

FIG. 12: $\left(\nabla_{r} \psi^{R}\right)_{m}$ versus $m$. Our results show an $m$-fall-off closest to $1 / \mathrm{m}^{4}$.

will focus on exploring the robustness of our technique against these more interesting cases.

\section{Acknowledgments}

We thank Roland Haas, Eric Poisson, and Bernard Whiting for helpful discussions pertaining to various aspects of this work. Development of the ideas in this manuscript began while one of us (S.D.) was at the Aspen Center for Physics during the 2005 summer workshop LISA Data: Analysis, Sources, and Science; we gratefully acknowledge the Center and the workshop organizers for their support and kind hospitality. These ideas were further developed at the eighth, ninth and tenth Annual Capra meetings at the Rutherford Appleton Laboratory Oxford (2005), University of Wisconsin in Milwaukee (2006) and University of Alabama in Huntsville (2007), respectively. And we are grateful to the organizers of these valuable workshops. The authors also acknowledge the University of Florida High-Performance Computing Center (URL: http://hpc.ufl.edu) and the Institute for Fundamental Theory (URL: http://www.phys.ufl.edu/ift) for providing computational resources and support that have contributed to the research results reported in this paper. This work was supported in part by the National Science Foundation, award No. PHY-0555484.

\section{APPENDIX A: APPROXIMATE SINGULAR FIELD IN THZ-COORDINATES}

A special coordinate system developed by Thorne and Hartle [20] and by Zhang [21] is particularly useful for self-force analyses. These THZ coordinates $(t, x, y, z)$ are defined in a neighborhood of a geodesic of a vacuum spacetime and are locally inertial, harmonic and Minkowskii-like, and centered on the geodesic with $t$ measuring the proper time along the geodesic. In these special coordinates our expression for the approximate singular field $\tilde{\psi}_{S}=q / \sqrt{x^{2}+y^{2}+z^{2}}$ is quite simple. But, for the case of a point charge in a circular orbit about a Schwarzschild black hole this simplicity of $\psi^{S}$ belies the hidden complexity of the coordinate transformation between the Schwarzschild coordinates $\left(t_{s}, r, \theta, \phi\right)$ and the THZ coordinates.

The full coordinate transformation, which we use for our analysis in the main body of this paper may be found in Eqs. (B1)-(B9) of Ref. [9]. Below we give only an abbreviated form of these equations to give a sense of how the coordinate transformation is implemented. The formulae below give $\bar{x}, \bar{y}, \bar{z}$ and $\bar{t}$ as smooth functions of the Schwarzschild $r, \theta, \phi$ and $t_{s}$. We can then define a function $\bar{\rho}=\sqrt{\bar{x}^{2}+\bar{y}^{2}+\bar{z}^{2}}$ which has the property that

$$
\nabla^{a} \nabla_{a}(1 / \bar{\rho})=-4 \pi \delta(\vec{x})+O(1 / \bar{\rho}) .
$$

If we had used $q / \bar{\rho}$ as the approximation $\tilde{\psi}_{s}$ for the singular field then the effective source for the regular field $\psi^{R}$ in the vicinity of the point charge would be singular,

$$
\bar{S}_{\mathrm{eff}}=-\nabla^{a} \nabla_{b}(q / \bar{\rho})-4 \pi q \delta(\vec{x})=O(1 / \bar{\rho}),
$$

rather than $O(\rho)$, which is the case for the effective source which we actually use as described in Eq. (10).

The coordinates which lead to $\bar{\rho}$ are now given for a circular geodesic of the Schwarzschild geometry at Schwarzschild radius $R$ : We first define two useful functions

$$
\begin{aligned}
\tilde{x}= & \frac{\left[r \sin \theta \cos \left(\phi-\Omega t_{\mathrm{s}}\right)-R\right]}{(1-2 M / R)^{1 / 2}}+\frac{M}{R^{2}(1-2 M / R)^{1 / 2}} \\
\times & {\left[\frac{(r-R)^{2}}{2(1-2 M / R)}+R^{2} \sin ^{2} \theta \sin ^{2}\left(\phi-\Omega t_{s}\right)+R^{2} \cos ^{2} \theta\right] } \\
& +O\left(\rho^{3}\right)
\end{aligned}
$$

and

$$
\tilde{y}=r \sin \theta \sin \left(\phi-\Omega t_{\mathrm{s}}\right)\left(\frac{R-2 M}{R-3 M}\right)^{1 / 2}+O\left(\rho^{3}\right) \text {. }
$$

The $O\left(\rho^{3}\right)$ terms indicate that these (and the formulae below) could be modified by the addition of arbitrary $O\left(\rho^{3}\right)$ terms without necessarily changing the usefulness of these coordinates.

In terms of these two functions, the THZ coordinates $(\bar{t}, \bar{x}, \bar{y}, \bar{z})$ are

$$
\bar{x}=\tilde{x} \cos \left(\Omega^{\dagger} t_{\mathrm{s}}\right)-\tilde{y} \sin \left(\Omega^{\dagger} t_{\mathrm{s}}\right)
$$


and

$$
\bar{y}=\tilde{x} \sin \left(\Omega^{\dagger} t_{\mathrm{s}}\right)+\tilde{y} \cos \left(\Omega^{\dagger} t_{\mathrm{s}}\right)
$$

where $\Omega^{\dagger}=\Omega \sqrt{1-3 M / R}$, along with

$$
\bar{z}=r \cos (\theta)+O\left(\rho^{3}\right)
$$

and

$$
\begin{aligned}
\bar{t}=t_{\mathrm{s}}(1 & -3 M / R)^{1 / 2} \\
& -\frac{r \Omega R \sin \theta \sin \left(\phi-\Omega t_{s}\right)}{R-3 M}+O\left(\rho^{3}\right)
\end{aligned}
$$

The set of functions $(\bar{t}, \tilde{x}, \tilde{y}, \bar{z})$ forms a non-inertial coordinate system that co-rotates with the particle in the sense that the $\tilde{x}$ axis always lines up the center of the black hole and the center of the particle, the $\tilde{y}$ axis is always tangent to the spatially circular orbit, and the $\bar{z}$ axis is always orthogonal to the orbital plane.

The THZ coordinates $(\bar{t}, \bar{x}, \bar{y}, \bar{z})$ are locally inertial and non-rotating in the vicinity of the charge, but these same coordinates appear to be rotating when viewed far from the charge as a consequence of Thomas precession as revealed in the $\Omega^{\dagger} t_{\mathrm{s}}$ dependence in Eqs. (A5) and (A6) above.

The coordinates $(\bar{t}, \bar{x}, \bar{y}, \bar{z})$ given above are said to be second order THZ coordinates and differ from the actual fourth order ones used in the main body of this paper by the replacement of the $O\left(\rho^{3}\right)$ terms appearing above by specific terms which scale as $\rho^{3}$ and $\rho^{4}[9]$ and leave the undetermined parts of the THZ coordinates being $O\left(\rho^{5}\right)$.

\section{APPENDIX B: CONVERGENCE FACTOR}

An $n$ th-order evolution code is one for which $\psi=$ $\psi_{N}(h)+\left(\epsilon^{(n)}(\xi)\right) h^{n}$, where $\psi_{N}(h)$ is the numerical solution at resolution $h$, and $\epsilon^{(n)}(\xi)$ is some unknown error function or order $\approx 1$. Consider three resolutions $h, 2 h$, 4h. This then leads to

$$
\begin{aligned}
& \psi=\psi_{N}(h)+\left(\epsilon^{(n)}(\xi)\right) h^{n} \\
& \psi=\psi_{N}(2 h)+\left(\epsilon^{(n)}(\xi)\right)(2 h)^{n} \\
& \psi=\psi_{N}(4 h)+\left(\epsilon^{(n)}(\xi)\right)(4 h)^{n}
\end{aligned}
$$

Thus,

$$
\frac{\left|\psi_{N}(4 h)-\psi_{N}(2 h)\right|}{\left|\psi_{N}(2 h)-\psi_{N}(h)\right|}=\left|\epsilon^{(n)}(\xi)\right| 2^{n},
$$

and so

$$
\begin{aligned}
n=\log & \left|\frac{\psi_{N}(4 h)-\psi_{N}(2 h)}{\psi_{N}(2 h)-\psi_{N}(h)}\right| / \log (2) \\
& +\log \left|\epsilon^{(n)}(\xi)\right| / \log (2) .
\end{aligned}
$$

[1] T. Regge and J. A. Wheeler, Phys. Rev. 108, 1063 (1957).

[2] F. J. Zerilli, Phys. Rev. D 2, 2141 (1970).

[3] S. A. Teukolsky, Astrophys. J. 185, 635 (1973).

[4] L. Barack and A. Ori, Phys. Rev. D 61, 061502 (2000), http://arxiv.org/abs/gr-qc/9912010.

[5] L. Barack, Y. Mino, H. Nakano, A. Ori, and M. Sasaki, Phys. Rev. Lett. 88, 091101 (2002).

[6] Y. Mino, H. Nakano, and M. Sasaki, Prog. Theor. Phys. 108, 1039 (2002), http://arxiv.org/abs/gr-qc/0111074.

[7] C. O. Lousto, Phys. Rev. Lett. 84, 5251 (2000).

[8] L. M. Burko, Phys. Rev. Lett. 84, 4529 (2000).

[9] S. Detweiler, E. Messaritaki, and B.F. Whiting, Phys. Rev. D 67, 104016 (2003), http://arxiv.org/abs/grqc/0205079.

[10] L. M. Diaz-Rivera, E. Messaritaki, B. Whiting, and S. Detweiler, Phys. Rev. D 70, 124018 (2004), http://arxiv.org/abs/gr-qc/0410011.

[11] W. Hikida, S. Jhingan, H. Nakano, N. Sago, M. Sasaki, and T. Tanaka, Prog. Theor. Phys. 111, 821 (2004), http://arxiv.org/abs/gr-qc/0308068.

[12] W. Hikida, S. Jhingan, H. Nakano, N. Sago, M. Sasaki, and T. Tanaka, Prog. Theor. Phys. 113, 283 (2005), http://arxiv.org/abs/gr-qc/0410115.

[13] R. Haas and E. Poisson, Physical Review D 74, 044009 (2006), http://arXiv.org/abs/gr-qc/0605077.

[14] R. Haas, Phys. Rev. D 75, 124011 (2007), http://arxiv.org/abs/gr-qc/0704.0797.

[15] L. Barack and N. Sago, Phys. Rev. D 75, 064021 (2007), http://arxiv.org/abs/gr-qc/0701069.

[16] B.S. DeWitt and R.W. Brehme, Ann. Phys. 9, 220 (1960).

[17] Y. Mino, M. Sasaki, T. Tanaka, Phys. Rev. D 55, 3457 (1997), http://arxiv.org/abs/gr-qc/9606018.

[18] T.C. Quinn and R.M. Wald, Phys. Rev. D 56, 3381 (1997), http://arxiv.org/abs/gr-qc/9610053.

[19] S. Detweiler and B.F. Whiting, Phys. Rev. D 67, 024025 (2003), http://arxiv.org/abs/gr-qc/0202086.

[20] K. S. Thorne and J. B. Hartle, Phys. Rev. D 31, 1815 (1985).

[21] X.-H. Zhang, Phys. Rev. D 34, 991 (1986).

[22] S. Detweiler, Phys. Rev. Lett. 86, 1931 (2001), http://arXiv.org/abs/grqc/0011039.

[23] S. Detweiler, Class. Quantum Grav. 22, S681 (2005), http://arxiv.org/abs/gr-qc/0501004.

[24] W. H. Press, S. A. Teukolsky, W. T. Vetterling, and B. P. Flannery, Numerical Recipes in C: The Art of Scientific Computing (Cambridge University Press, Cambridge, 1992), 2nd ed.

[25] C.O. Lousto and R.H. Price, Phys. Rev. D 56, 6439 (1997), http://arxiv.org/abs/gr-qc/9705071.

[26] C.O. Lousto, Class. Quantum Grav. 22, S543 (2005), http://arxiv.org/abs/gr-qc/0503001.

[27] R. Lopez-Aleman, G. Khanna, and J. Pullin, 
Classical and Quantum Gravity 20, 3259 (2003), http://arxiv.org/abs/gr-qc/0303054.

[28] L. M. Burko and G. Khanna, Europhysics Letters 78, 60005 (2007), http://arXiv.org/abs/gr-qc/0609002.

[29] P. A. Sundararajan, G. Khanna, and S. A. Hughes, Phys. Rev. D 76, 104005 (2007).

[30] A. Pound and E. Poisson (2007), http://arXiv.org/abs/0708.3037 [gr-qc].

[31] A. Pound and E. Poisson (2007),
http://arXiv.org/abs/0708.3033 [gr-qc].

[32] L. Barack and D. Golbourn, Phys. Rev. D 76, 044020 (2007), http://arxiv.org/abs/gr-qc/0705.3620.

[33] L. Barack, D. A. Golbourn, and N. Sago (2007), http://arXiv.org/abs/0709.4588 [gr-qc].

[34] With $\rho^{2} \equiv x^{2}+y^{2}+z^{2}$, a function which is $O\left(\rho^{n}\right)$ as $\rho \rightarrow 0$, is at least $C^{n-1}$ where $\rho=0$. 